\title{
A Concept of Information Model Referring to the Big Bang Theory
}

\author{
Andrzej Kaczmarczyk \\ Modeling and Simulation Department, Institute of Mathematical Machines, Warsaw, 02-078, Poland
}

\begin{abstract}
A model of information considered as an attribute of the matter, having Big bang theory as a reference frame, is presented in the article. According to the model, information defined as properties of material objects, manifests itself in a dual information space spread by Big Bang over the arising material universe. Both conjugated spaces are represented by binary hyper-cubes (Hamming spaces) in which space's elements are identified by means of binary code. One of the conjugated spaces is property-space, the second one object-space. The object-space reflects real objects, and includes also imaginary objects generated as a result of combinations of properties of the universe. In this respect the model has an affinity with Platonic Theory of Forms.
\end{abstract}

Keywords Information Model, Dual Information Space, Big Bang

\section{Introduction}

Since 1970s the information concept has played significant role in the "Theory of the Universe" thanks to development of digital mechanics, and even more: digital philosophy. Digital view of the universe consists in assumption that all processes can be "viewed as computation", and the universe can be depicted by means of automata theory as a cellular automaton functioning by right of quantum computer[1-3]. So, the "digital" concept of information is focused on the aspect of its transmission, because in the digital approach "structure of the multiverse is determined by information flow"[4]. According to the classification of information concepts proposed in[5], the transmission aspect is the second of three possible aspects. First of them, the most fundamental because relates to the question what exactly does flow, is the aspect of "information given", called "potential" or "structural" one.

The subject of this article is an information model related to the first aspect - structural information of the universe with the Big Bang theory as a reference frame. In this Big Bang Information (BBI) model information is defined as matter's attribute, and - after the digital concept - the assumption on discrete character of the matter is adopted.

The third aspect of information discussed in[5] is "the view of the receiver", so of information processed by a receiver that produces "actual" information having a meaning. This aspect is not considered in the BBI model that is

* Corresponding author:

kaczmarczyka@yahoo.com (Andrzej Kaczmarczyk)

Published online at http://journal.sapub.org/ijis

Copyright (C) 2012 Scientific \& Academic Publishing. All Rights Reserved limited now to the primary information accessible for cybernetic systems embodied both by live and artificial beings by which can be taken, processed, sent, and used.

\section{BBI Model}

Let's assume that as a result of the Big Bang, at the very beginning only one Object of Material Universe (OMU) appeared, and that subsequent expansion of Universe consisted in appearance of next OMUs of discrete character. Information as an attribute of the matter manifests itself in properties of OMUs: in the BBI model properties of an OMU are considered structural information attributed to it.

Thus, in the BBI model, a set representing Material Universe, with its members-elements representing OMUs is considered. In the set theory, a property that characterizes an element of a set is determined by a subset of elements, including this one under consideration, which satisfy the property. Properties of space-time can be an expressive example. OMUs appearing in different locations in space form subsets determining particular values of space coordinates; similarly OMUs appearing in different moments of time form time-marking subsets.

Therefore, all properties characterizing the Universe, meaning the whole information attributed to it, is determined by the power set - the set of all subsets - of a fundamental set of all OMUs. Numerically, if the fundamental set includes $n$ OMUs-elements, then it has $N=2^{n}$ subsets-properties.

Let's consider examples of simple sets-Universes.

Empty set $n=0: \varnothing$

Its subsets: $\varnothing$

Number of subsets-properties: $N=2^{0}=1$ 
One-element set $n=1:\{a\}$

Its subsets: $\varnothing ;\{a\}$

Number of subsets-properties: $N=2^{1}=2$

Two-element set $n=2:\{a, b\}$

Its subsets: $\varnothing ;\{a\} ;\{b\} ;\{a, b\}$

Number of subsets-properties: $N=2^{2}=4$

Three-element set $n=3:\{a, b, c\}$

Its subsets: $\varnothing ;\{a\} ;\{b\} ;\{c\} ;\{a, b\} ;\{a, c\} ;\{b, c\} ;\{a, b, c\}$

Number of subsets-properties: $N=2^{3}=8$

The number $N$ describes the amount of information attributed to the Universe. It is worth to note that if this amount is expressed in bits, it equals the number of elements of a set-Universe:

$$
I_{\text {bit }}=\log _{2} N=\log _{2} 2^{n}=n
$$

where $I_{b i t}$ is amount of information in bits.

The key postulation of the BBI model affirms that the Big Bang explosion giving birth to the Material Universe simultaneously spreads an information space over it. This information space, characteristic of which should meet all so far revealed features of information attributed to the Universe, is of a dual nature, grouping of two conjugated spaces: property-space and object-space.

In the BBI model these spaces are formally represented by binary hyper-cubes, known also as Hamming spaces, in which space's elements - properties and objects respectively - are identified by means of binary code.

The property-space is represented by $n$-dimensional hyper-cube - i.e. $n$-bit binary cube. An example of the property-space for previously discussed three-element Universe is presented on the Fig. 1 .

\begin{tabular}{|c|c|c|c|}
\hline OBJECT & $a$ & $b$ & $c$ \\
\hline$\varnothing \quad$ & 0 & 0 & 0 \\
\hline$\{a\}$ & 1 & 0 & 0 \\
\hline$\{b\}$ & 0 & 1 & 0 \\
\hline$\{c\}$ & 0 & 0 & 1 \\
\hline$\{a, b\}$ & 1 & 1 & 0 \\
\hline$\{a, c\}$ & 1 & 0 & 1 \\
\hline$\{b, c\}$ & 0 & 1 & 1 \\
\hline$\{a, b, c\}$ & 1 & 1 & 1 \\
\hline
\end{tabular}

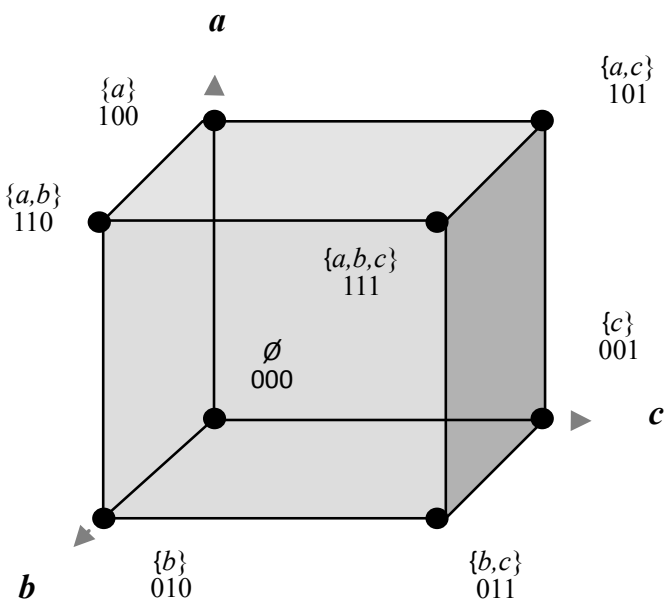

Figure 1. Information property-space over three-element Universe
Elements $a, b$ and $c$, being objects of the Universe, serve as coordinates of the space. They become coding bits enabling presentation of properties in binary code in which values 0 and 1 have logical meaning "no" and "yes". So, e.g. the property $\{b, c\}$ is coded as $011-$ meaning " $a-$ no", " $b-$ yes", "c - yes". Graphically, properties of the Universe are represented by corners of the cube.

The object-space also is represented by binary hyper-cube, but this time inversely: properties are coordinates and coding bits, and objects are coded entities symbolized by cube corners. Therefore, the hyper-cube is $N$ - dimensional in this case. The example of previous three-element Universe, giving 8-dimensional hyper-cube, is to complex for graphical presentation. Thus, simpler two-element Universe will be examined as an example. The object-space over this Universe is presented on Fig.2. However, even in this simpler case we deal with 4-dimensional hyper-cube, and it is necessary to present graphically the cube by two its 3 -dimensional cross-sections. One of them is made for the value 0 of $\varnothing$ property-coordinate, the second for its value 1 .

The key finding brought by object-space examination is appearance of imaginary objects in it. The BBI model shows that the information space not only reflects real OMUs, but also gives birth to imaginary objects generated as a result of all possible combinations of properties of the Material Universe. Imaginary objects having inconsistent properties are only thinkable, but impossible to be brought to being. For example, a square with all points of its periphery equally distant from the square's centre of symmetry is thinkable ("postulatable"), but cannot be brought to existence. One can suppose that other imaginary objects, such ones which are not inconsistent, can be designed - not out of the question that by rational beings which emerge in the process of cosmic evolution.

According to the Big Bang theory, shortly after explosion, a phase of cosmic inflation ensued, during which cosmos expanded by a factor of million trillion trillion in a tiny fraction of a second ${ }^{1}$. So, in the BBI model it should be admitted that the number $n$ of OMUs, thereby the amount of information attributed to the Universe, quickly approaches infinity in constructivist sense (infinity in constructivist sense is understood as a multitude so great that is practically uncountable) $)^{2}$.

As it was stated previously, in the BBI model properties of an OMU are considered structural information attributed to it. It's easy to notice, observing the examples of simple

\footnotetext{
${ }^{1}$ Exactly speaking, inflation process lasted from $10^{-36}$ seconds after the explosion to $10^{-32}$ seconds. In this period, exponential expansion increased volume of the early cosmos by a factor of at least $10^{78}$ - it means that in this time cosmos volume changed from smaller than an atom to around 100 million light years across.

${ }^{2}$ In 1938 British astronomer Arthur Eddington calculated the number of protons - according to his opinion equal the number of electrons - in the observable universe. This number $N_{E d d}$ is being updated in accordance with new cosmological findings, but can be estimated as $10^{80}$. If one considered Eddington number as number of OMUs, the amount of information of the BBI model's universe would equal $10^{80}$ bits.
} 
sets-Universes, that each element-OMU has a half of all properties of the Universe; i.e. a half of structural information of the Material Universe is attributed to each of its elements (it can be proved for $n$-element set). Thereby, also information attributed to each OMU approaches infinity during cosmic inflation.

\begin{tabular}{|c|c|c|c|c|}
\hline PROPERTY & $\varnothing$ & $\{\mathrm{a}\}$ & $\{\mathrm{b}\}$ & $\{\mathrm{a}, \mathrm{b}\}$ \\
\hline OBJECT & 0 & 0 & 0 & 0 \\
\hline Imaginary object & 1 & 0 & 0 & 0 \\
\hline Imaginary object & 0 & 1 & 0 & 0 \\
\hline Imaginary object & 0 & 0 & 1 & 0 \\
\hline Imaginary object & 0 & 0 & 0 & 1 \\
\hline Imaginary object & 1 & 1 & 0 & 0 \\
\hline Imaginary object & 1 & 0 & 1 & 0 \\
\hline Imaginary object & 1 & 0 & 0 & 1 \\
\hline Imaginary object & 0 & 1 & 1 & 0 \\
\hline Imaginary object & 0 & 1 & 0 & 1 \\
\hline Real object a & 0 & 0 & 1 & 1 \\
\hline Real object b & 1 & 1 & 1 & 0 \\
\hline Imaginary object & 1 & 1 & 0 & 1 \\
\hline Imaginary object & 0 & 1 & 1 & 1 \\
\hline Imaginary object & 1 & 0 & 1 & 1 \\
\hline Imaginary object & 1 & 1 & 1 & 1 \\
\hline Imaginary object & & & &
\end{tabular}

$\{a\}$

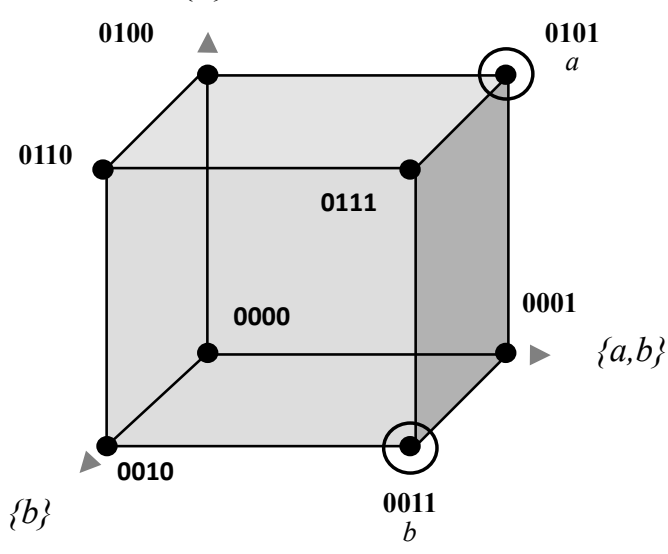

Value 0 of the coordinate $\varnothing$

$$
\{a\}
$$

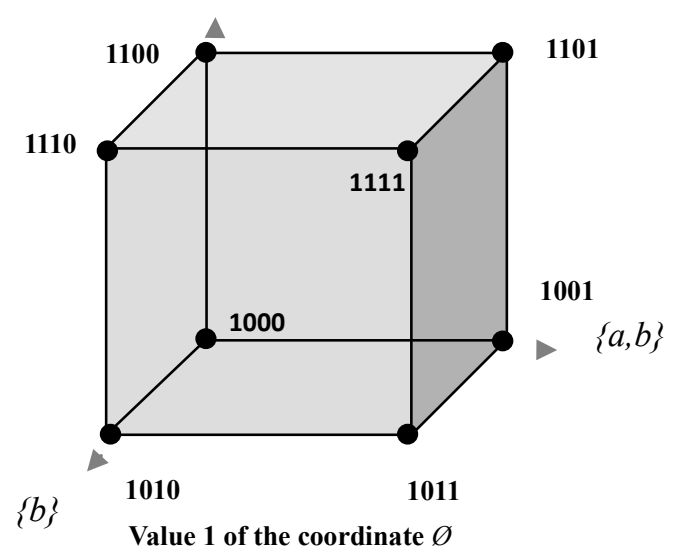

Figure 2. Information object-space over two-element Universe

\section{Summary and Conclusions}

In the BBI model, expanding Material Universe being result of the Big Bang explosion is represented by increasing discrete set of material objects. Properties of these objects are considered structural information attributed to them. Together with giving birth to the Material Universe, the Big Bang spreads over the Universe an information space which is of a dual nature, i.e. it groups two conjugated spaces: property-space and object-space. Both spaces are formally represented in the BBI model by binary hyper-cubes - Hamming spaces - in which space's elements are identified by means of binary code. The object space not only reflects real objects, but also includes imaginary objects generated as a result of all possible combinations of properties of the Material Universe. The amount of information attributed to the set-Universe, expressed in bits, equals the numbers of its elements i.e. the number of real objects of the Material Universe. During the period of cosmic inflation, when cosmos is subjected to extremely rapid exponential expansion, the number of objects - thereby the amount of information - quickly approach infinity.

From the philosophical point of view, the BBI model can be interpreted as certain modification of Platonic Theory of Forms. According to Plato, perfect archetypes - Forms or Ideas (gr. $l \delta \varepsilon^{\prime} \alpha$ ) - are true eternal entities, and material objects only their imperfect copies-reflections. Inversely than in Plato, in the BBI model objects of the Material Universe are original, and information as materia prima of Ideas is derivative. However "Ideas", in the form of imaginary objects, are situated in the information object-space, and can be brought to rise by rational beings.

Further work on the BBI model can relate to two issues. One of them is more profound and detailed characterization of the mathematical substance of the model, analysis and interpretation of its mathematical properties, probably with application of operations on infinite numbers. The second issue is introduction into the model more complex assumptions about the Material Universe, closer to its current cosmological characterization.

\section{REFERENCES}

[1] Zuse K. (1969). Rechnender Raum. Braunschweig: Friedrich Vieweg \& Sohn.

[2] Fredkin E. (2003). An Introduction to Digital Philosophy. International Journal of Theoretical Physics, Vol. 42, No. 2.

[3] Wolfram S. (2002). A New Kind of Science. Wolfram Media, Inc.

[4] Deutch D. (2001). The Structure of the Multiverse. arXiv:quant-ph/0104033.

[5] Hofkirchner, W. (2009). How to achieve a unified theory of information. triple $\mathrm{C}, 7(2)$ 